\title{
L. Mansi, E. Lopci, V. Cuccurullo and A. Chiti (eds): Clinical Nuclear Medicine in Pediatrics
}

\author{
Springer International Publishing, Switzerland, 2016, ISBN 978-3-319-21370-5
}

\author{
Angelika Bischof Delaloye ${ }^{1}$
}

Published online: 26 February 2016

(C) Springer-Verlag Berlin Heidelberg 2016

As we all know, children are not just little adults. Therefore, paediatric nuclear medicine is not restricted to the use of radiopharmaceuticals with lower activity. This book emphasizes the value of nuclear medicine in children and covers all essential aspects of nuclear imaging and therapy in 18 chapters (380 pages). More interestingly, it develops in detail the specific aspects of dealing with children. From the first chapter the importance of understanding diagnostic nuclear imaging in children in its full dimension, covering medical, psychological, social and technical aspects, is underlined. The authors emphasize the importance of a specific approach to the management of children (including sedation and anaesthesia, and methods of pain and stress reduction), the need for an appropriately designed patient-friendly facility and also the need for alternative diagnostic methods.

After a chapter on the great potential of PET/MRI, current issues in paediatric molecular radiotherapy are addressed, followed by a comprehensive presentation on radiation risks in children. An extensive chapter is devoted to paediatric nuclear medicine in the acute setting. This chapter might be used not only to raise the awareness of clinicians concerning the possibilities offered by nuclear medicine techniques in the differential diagnosis of acute symptoms but also to convince administrators of the necessity of having nuclear medicine services available 24 hours a day, 7 days a week. The following five chapters address the role of paediatric nuclear medicine in assessing pathological conditions of various organ systems (cardiology, endocrinology, bone, gastrointestinal tract and nephrourology).

Angelika Bischof Delaloye

Angelika.BischofDelaloye@chuv.ch

1 Faculty of Medicine and Biology, University of Lausanne, Lausanne, Switzerland
The second half of the book is devoted to cancer in children. After a general chapter written by paediatric oncologists on paediatric cancers, including epidemiology, clinical presentation, pathology, molecular characterization, genetic anomalies, diagnostic workup, treatment and follow-up, the contributions of nuclear medicine in the management of the most frequent types (lymphoma, neuroblastoma, sarcoma, cerebral tumours, thyroid cancer and other neoplasms) are presented in separate chapters. Finally, an outlook on diagnostic paediatric nuclear medicine in Eastern European countries using the example of Russia concludes the book.

The editors assigned the task of writing the various chapters to well-chosen Italian and international authors. Despite their great number a certain unity in the chapters has been maintained. There are some repetitions but is repetition not the mother of all learning?

All chapters are comprehensive and well structured, covering clinical and technical (radiopharmaceuticals, instrumentation) aspects, interpretation and pitfalls; a table of contents precedes each of them, thus facilitating quick search for particular information. The book is pleasant to read with numerous illustrations that are well chosen and of excellent quality. This makes it an outstanding tool for teaching students, technologists and nuclear medicine residents. In nuclear medicine services where paediatric patients are less often seen than in dedicated units - and even in the latter - this book should be at hand for staff members, above all physicians, technologists and nurses. Relevant information can easily be found to refresh the memory, eliminate doubts or review study protocols. In this context, it might perhaps have been helpful to add a list of relevant major international guidelines with their references and links in addition to citing them. For all those who would like to dig deeper into the matter, extensive lists of references at the end of each chapter cover the literature on paediatric nuclear medicine and related clinical and basic science. 